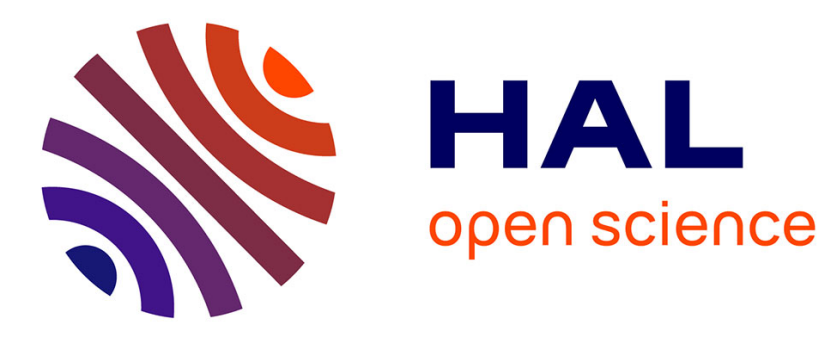

\title{
Bio-inspired Landing Approaches and Their Potential Use On Extraterrestrial Bodies
}

\author{
Thibaut Raharijaoana, Guillaume Sabiron, Stéphane Viollet, Nicolas
}

Franceschini, Franck Ruffier

\section{- To cite this version:}

Thibaut Raharijaoana, Guillaume Sabiron, Stéphane Viollet, Nicolas Franceschini, Franck Ruffier. Bio-inspired Landing Approaches and Their Potential Use On Extraterrestrial Bodies. Springer Verlag Berlin Heidelberg. Viorel Badescu, Springer Verlag Berlin Heidelberg, pp.221-246, 2013, 9783642392436. hal-00858087

\section{HAL Id: hal-00858087 \\ https://hal.inria.fr/hal-00858087}

Submitted on 4 Sep 2013

HAL is a multi-disciplinary open access archive for the deposit and dissemination of scientific research documents, whether they are published or not. The documents may come from teaching and research institutions in France or abroad, or from public or private research centers.
L'archive ouverte pluridisciplinaire HAL, est destinée au dépôt et à la diffusion de documents scientifiques de niveau recherche, publiés ou non, émanant des établissements d'enseignement et de recherche français ou étrangers, des laboratoires publics ou privés. 


\title{
Bio-inspired Landing Approaches and Their Potential Use On Extraterrestrial Bodies
}

\author{
Thibaut Raharijaona, Guillaume Sabiron, \\ Stéphane Viollet, Nicolas Franceschini \\ and Franck Ruffier
}

\begin{abstract}
Automatic landing on extraterrestrial bodies is still a challenging and hazardous task. Here we propose a new type of autopilot designed to solve landing problems, which is based on neurophysiological, behavioral, and biorobotic findings on flying insects. Flying insects excel in optic flow sensing techniques and cope with highly parallel data at a low energy and computational cost using lightweight dedicated motion processing circuits. In the first part of this paper, we present our biomimetic approach in the context of a lunar landing scenario, assuming a 2-degree-of-freedom spacecraft approaching the moon, which is simulated with the PANGU software. The autopilot we propose relies only on optic flow (OF) and inertial measurements, and aims at regulating the $\mathrm{OF}$ generated during the landing approach, by means of a feedback control system whose sensor is an OF sensor. We put forward an estimation method based on a two-sensor setup to accurately estimate the orientation of the lander's velocity vector, which is mandatory to control the lander's pitch in a near optimal way with respect to the fuel consumption. In the second part, we present a lightweight Visual Motion Sensor (VMS) which draws on the results of neurophysiological studies on the insect visual system. The VMS was able to perform local 1-D angular speed measurements in the range $1.5^{\circ} / \mathrm{s}-25^{\circ} \mathrm{s}$. The sensor was mounted on an $80 \mathrm{~kg}$ unmanned helicopter and test-flown outdoors over various fields. The OF measured on-

Thibaut Raharijaona, Guillaume Sabiron, Stéphane Viollet, Nicolas Franceschini and Franck Ruffier

Aix-Marseille University, CNRS, Institute of Movement Science, Biorobotics Dept., UMR7287, 13288, Marseille, France

Tel.: +33-4-91828365

E-mail: \{thibaut.raharijaona,guillaume.sabiron,stephane.viollet\}@univ-amu.fr

E-mail: \{nicolas.franceschini,franck.ruffier\}@univ-amu.fr

Guillaume Sabiron

French Aerospace Lab (ONERA, Systems Control and Flight Dynamics -DCSD-), 31055

Toulouse, France

Tel.: +33-4-91828366

E-mail: guillaume.sabiron@onera.fr
\end{abstract}


board was shown to match the ground-truth optic flow despite the dramatic disturbances and vibrations experienced by the sensor.

\section{Nomenclature}

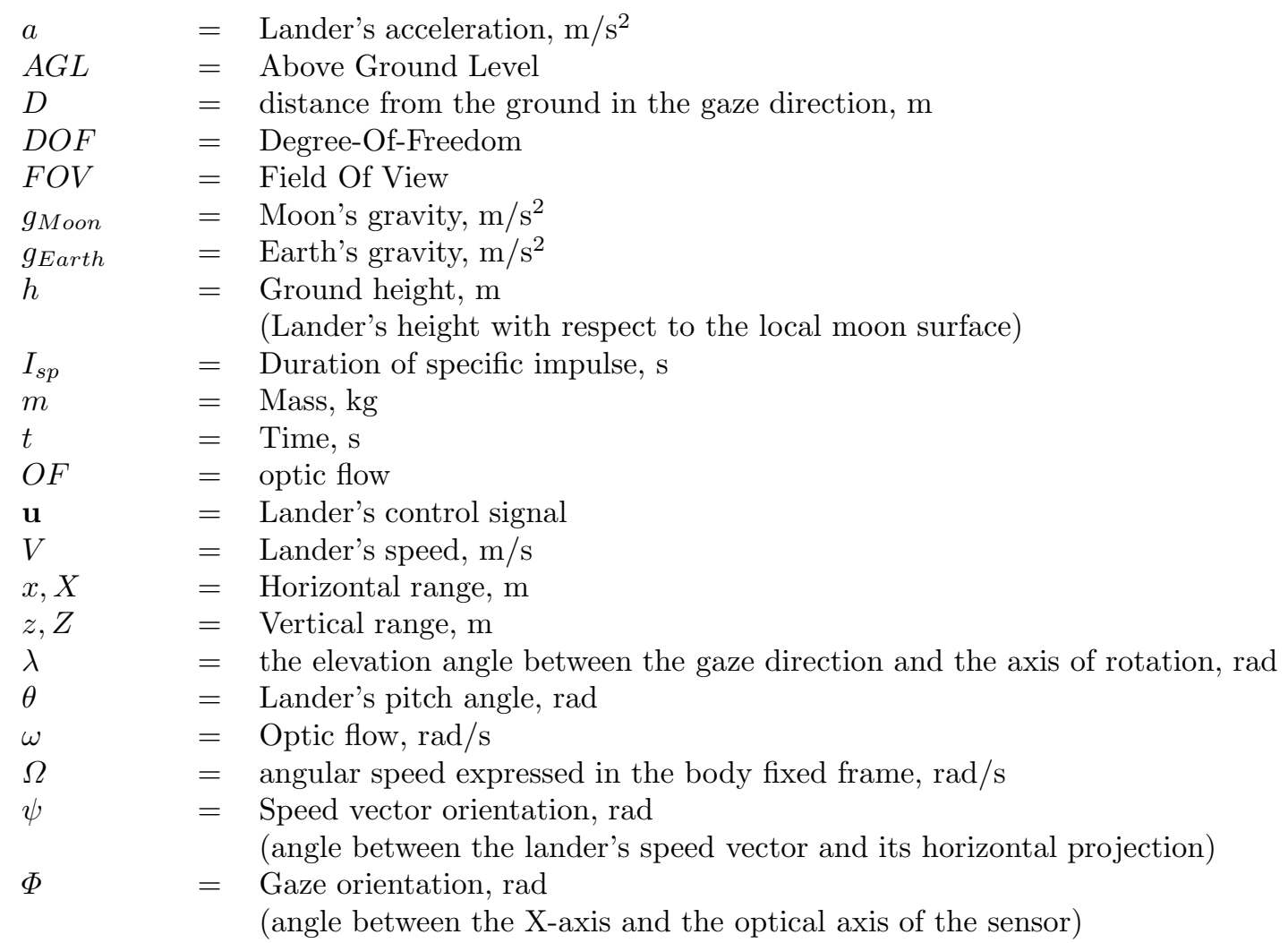

$\begin{array}{lll}\text { Subscripts } & & \\ \text { grd } & = & \text { ground } \\ l d r & = & \text { lander } \\ t h & = & \text { thruster } \\ \text { trh } & = & \text { truth } \\ \text { ref } & = & \text { reference } \\ R & = & \text { rotational } \\ T & = & \text { translational } \\ f & = & \text { final } \\ 0 & = & \text { initial }\end{array}$




\section{Introduction}

Landing on asteroids and extraterrestrial bodies is a critical stage for future exploration missions. Safe and soft landing on asteroids will be required even though the task is way harder than on the Earth due to the small size, irregular shape and variable surface properties of asteroids, as well as the low gravity and negligible drag experienced by the spacecraft. Optical guidance and navigation for autonomous landing on small celestial bodies have been studied in the past years with a focus on the closed-loop guidance, navigation, and control (GNC) systems (De Lafontaine, 1992; Kawaguchi et al., 1999).

The complexity of the task is dramatically increased by the lack of atmosphere since absence of drag precludes the deployment of parachutes, in contrast with the situation on Mars (Braun and Manning, 2006) and other planets.

Added to this are the lack of reliable terrain and obstacle data bases and the lack of conventional sensing systems such as Global Positioning Systems (GPS) as well. On top of that, prohibitive latency in communication between the Earth and the extraterrestrial body obviously forces the landing to be made autonomously while requiring robust, reliable and powerless sensors. Precise GNC systems are indeed needed for safe and accurate landing in unprepared landing zones. Tough requirements in terms of embedded mass led us to design a very lightweight biologically inspired nonemissive optical sensor that is able to measure the optic flow $(\mathrm{OF})$, that is, the angular velocity (in $\% / s$ ) of the visual image sweeping backwards across the visual field.

Visual cues seem to be a promising way towards autonomous landing. Recently, several studies have shown various optical techniques such as LIDAR (LIght Detection And Ranging) techniques (Parkes and Silva, 2002; Parkes et al., 2003) or vision based navigation systems to estimate spacecraft position and velocity parameters (Roumeliotis et al., 2002; Frapard et al., 2002; Cheng and Ansar, 2005; Janschek et al., 2006; Trawny et al., 2007; Flandin et al., 2009; Mourikis et al., 2009; Shang and Palmer, 2009), to avoid obstacle (Strandmoe et al., 1999) or to control unmanned spacecraft (Valette et al., 2010; Izzo et al., 2011; Izzo and de Croon, 2011). In (Valette et al., 2010), the OF regulation principle (Ruffier and Franceschini, 2005) was applied to autonomous lunar landing problems using a feedback loop aimed at maintaining the ventral optic flow constant. The approach was tested in simulation experiments using the PANGU software (Planet and Asteroid Natural scene Generation Utility) developed for ESA (European Space Agency) by the University of Dundee (see (Parkes et al., 2004; Dubois-Matra et al., 2009) for more information), which is a tool used to simulate visual environment of planetary surfaces. In (Izzo et al., 2011), based on numerical simulations, optimal trajectories were calculated in terms of the duration of the landing phase or the fuel consumption while keeping the OF constant. In (Mahony et al., 2008), a fully OF-based visual servo control system was developed, in which a large visual field was combined with a centroid in order to estimate the direction of the speed vector in the case of simulated small aerial robotic vehicles. In the 
extended Kalman filter (EKF) method described in (Mourikis et al., 2009), both of the above visual approaches were combined with an inertial measurement unit, and accurate estimates of the lander's terrain-relative position, attitude, and velocity were obtained. In the approach presented here, we focus on $\mathrm{OF}$ based means to derive useful information such as the orientation of the spacecraft velocity vector.

Finding means of sensing the OF onboard unmanned aerial and terrestrial vehicles has been a key research topic during the last few decades. Several flight control systems based on OF cues have been constructed so far for performing hazardous tasks such as hovering and landing on a moving platform (Herisse et al., 2012), avoiding obstacles (Barrows and Neely, 2000; Griffiths et al., 2006; Beyeler et al., 2009), following terrain (Netter and Franceschini, 2002; Ruffier and Franceschini, 2003, 2004, 2005; Garratt and Chahl, 2008) and tracking a moving target (Viollet and Franceschini, 1999a; Kendoul et al., 2009; Kerhuel et al., 2012). Some of the studies quoted above were inspired by insects, the impressive flight behavior of which relies on the built-in abilities they have developed and improved over several hundred millions of years, despite their small size and limited neural resources.

Flies, in particular, are agile seeing creatures that navigate swiftly through "unprepared" environments, avoiding obstacles with little conventional avionics. Equipped with "only" about one million neurons and "only" 3000 pixels in each eye, the common housefly, for example, achieves 3D navigation, terrain and obstacle avoidance at an impressive 700 body-lengths per second. All this is achieved, surprisingly, without any connections of the animal to a super-computer and an external power supply. The impressive lightness of the processing system at work onboard a fly or a bee makes any roboticist turn pale once he/she realizes that these creatures actually display many of the behaviors that have been sought for in the field of autonomous robotics for the last 50 years: dynamic stabilization, 3D collision avoidance, tracking, docking, autonomous landing in uncharted landing zones, etc.

The front end of the fly visual system consists of a mosaic of facet lenslets (see left hand side in Fig. 1) each of which focuses light on a small group of photorececeptor cells (see right hand part in Fig. 1). Flies possess one of the most complex and best organized retinae in the animal kingdom. Each of their photoreceptor cells is more sensitive and reliable than any photomultiplier ever built, and the two central cells (R7 and R8) display up to four different spectral sensitivities, from near UV to red (Franceschini, 1985).

In the mid 1980's, we started designing a fly-inspired robot to demonstrate how an agent could possibly navigate in a complex environment on the basis of OF (Blanes, 1986; Pichon et al., 1989). The Robot-Fly (Fig. 2.A) was equipped with a widefield curved compound eye embedding an array of 114 fly-inspired Local Motion Sensors (LMS) (Franceschini et al., 1991, 1992, 1997).

The robot was equipped with a planar compound eye and a fly-inspired Elementary Motion Detector neuron's array (Pichon et al., 1989).

The sensor array was used to sense the OF generated during the robot's own locomotion among stationary objects. The 50-cm high "robot-mouche" 


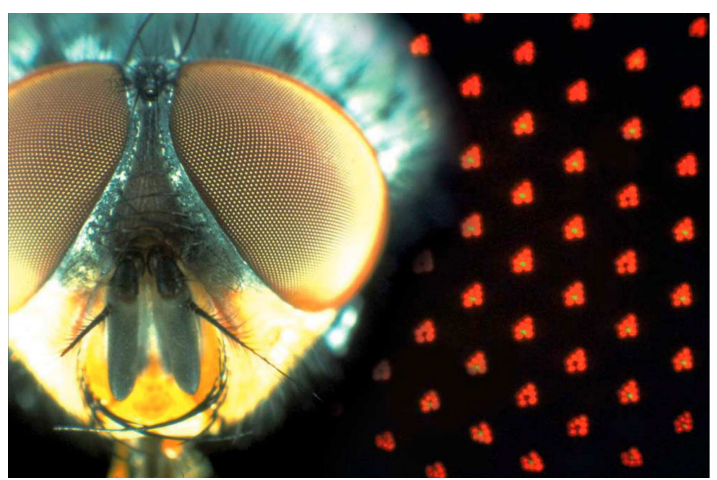

Fig. 1 Left: Head of the blowfly Calliphora erythrocephala (male) showing the two panoramic compound eyes with their facetted cornea. Right: Each ommatidium contains seven micrometer-sized photoreceptors in the focal plane of each facet-lens, as observed in vivo here (natural autofluorescence colors). Six outer receptors (R1-6) mediate motion vision and drive optic flow sensor neurons, while the two central cells (R7, prolonged by an R8 receptor not seen here) are in charge of color vision.. Figure from (Franceschini et al., 2010).

(Robot-Fly, in English) that we constructed in 1991 (see Fig. 2A) was the first OF-based, completely autonomous robot able to avoid contrasting obstacles encountered on its way, while traveling to its target at a relatively high speed $(50 \mathrm{~cm} / \mathrm{s})$ (Blanes, 1991; Franceschini et al., 1991, 1992, 1997). The Robot-Fly was primarily based on ethological findings on real flies, whose most common flight trajectories were shown to consist of straight flight sequences interspersed with rapid turns termed saccades (Collett and Land, 1975; Heisenberg and Wolf, 1984; Wagner, 1986), see also (Schilstra and Hateren, 1999; Tammero and Dickinson, 2002).

Based on the results of electrophysiological findings on fly motion detecting neurons obtained at our Laboratory (Franceschini, 1985; Franceschini et al., 1989), we developed a 2-pixel Local Motion Sensor (LMS) and proposed several versions of it over the years (Franceschini et al., 1992; Franceschini, 1999; Ruffier et al., 2003; Ruffier, 2004; Franceschini et al., 2007; Expert et al., 2011). The processing scheme at work in this sensor was introduced in (Blanes, 1986; Pichon et al., 1989), and later called the "facilitate-and-sample scheme" (Indiveri et al., 1996) or the time-of-travel scheme (Moeckel and Liu, 2007). Other vision-based systems have been used to measure the OF onboard UAVs (Unmanned Aerial Vehicles) (Green et al., 2004; Hrabar et al., 2005; Beyeler et al., 2009; Conroy et al., 2009) and in particular in the range experienced during lunar landing (Griffiths et al., 2006; Kendoul et al., 2009; Watanabe et al., 2009). Most of these visual systems were quite demanding in terms of their computational requirements and/or their weight or were not very well characterized, except for the optical mouse sensors (Beyeler et al., 2009), with which a standard error of approximately $\pm 5^{\circ} / \mathrm{s}$ around $25^{\circ} / \mathrm{s}$ was obtained in a $\pm 280^{\circ} / \mathrm{s}$ overall range. More recently we developed at the laboratory the concept of Visual Motion Sensor (VMS) by fusing the local measurement from several 2-pixel LMS to measure the 1-D OF more accurately and more 


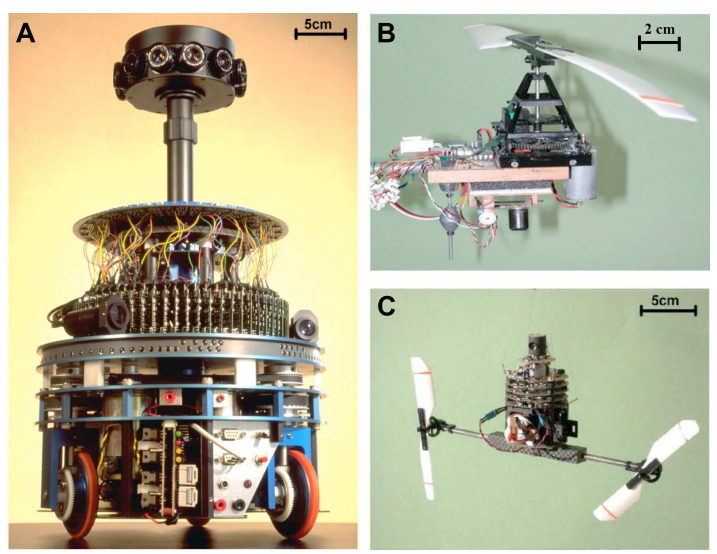

Fig. 2 Three of the visually-guided robots designed and constructed at the Laboratory on the basis of biological findings on visuomotor control in flies. (A) The 10-kg RobotFly ("robot-mouche" in French) incorporates the compound eye (visible at half-height) for obstacle avoidance, and a dorsal eye ("target seeker") for detecting the light source serving as a goal. This robot (height: $50 \mathrm{~cm}$; weight: $12 \mathrm{~kg}$ ) reacts to the OF generated by its own locomotion amongst obstacles (Franceschini et al., 1991, 1992, 1997). It is fully autonomous as regards its processing and power resources. (B) The robot OCTAVE (OF-based Control sysTem for Aerial VEhicles) is a 100-gram rotorcraft equipped with a 2-pixel ventral eye sensing the $\mathrm{OF}$ on the terrain below. This self-sustained aerial creature is tethered to a light rotating arm that allows only three degrees of freedom: forward and upward motion and pitch. The robot lifts itself and circles around a central pole at speeds up to $3 \mathrm{~m} / \mathrm{s}$. It ascends or descends depending on the ventral OF it measures (from (Ruffier and Franceschini, 2003, 2004, 2005). (C) The robot OSCAR (OF based Scanning sensor for the Control of Aerial Robots) is a 100-gram, twin-engined aircraft equipped with a two-pixel frontal visual system that relies on visual motion detection and on a microscanning process inspired by the fly (Franceschini and Chagneux, 1997). It is tethered to a 2-meter-long nylon wire secured to the ceiling of the laboratory. Vision and rate gyro signals combine onboard enabling OSCAR to fixate a target (a dark edge or a bar) with hyperacuity and to track it at angular speeds of up to $30^{\circ} / \mathrm{s}$ (Viollet and Franceschini, 1999b, 2001). Figure from (Franceschini et al., 2009).

frequently (Expert et al., 2012; Roubieu et al., 2011, 2012; Ruffier and Expert, 2012).

Few studies have been published so far, to our knowledge, in which OF systems were implemented and tested outdoors onboard an unmanned aircraft subject to vibrations, where the illuminance cannot be easily controlled (see (Barrows and Neely, 2000) in the case of linear 1-D motion sensors and see (Griffiths et al., 2006; Tchernykh et al., 2006; Garratt and Chahl, 2008; Kendoul et al., 2009) in that of 2-D OF sensors).

It therefore seemed to be worth testing the reliability of the present 1-D OF-based visual sensor on a platform that would experience conditions similar to those of a spacecraft in landing approach in terms of vibration dynamics and $\mathrm{OF}$ measurement range. To that aim, the sensor was embedded onboard a free-flying, unmanned helicopter and tested in terms of its resolution, accuracy and sensitivity. Particular efforts were made to adapt the sensor's measurement 
range $[1.5 / \mathrm{s}$ to $25 / \mathrm{s}]$ to the one a spacecraft would experience during a lunar landing approach (in the order of $[2 / \mathrm{s}-6 / \mathrm{s}]$ ).

The intended control strategy, the reference descent trajectory and the basic equations of the spacecraft dynamics are described in Sec. 2 with particular reference to lunar landing approaches. Neuromorphic principles are applied to monitoring and processing the OF during an autonomous vision-based extraterrestrial landing scenario. OF measurements allowing the estimation of the orientation of the velocity vector are underlined. Section 3 gives a brief description of the new 1-D visual motion device, outlines the processing algorithm and the implemented electro-optical assembly. Results of the tests performed on the airborne visual sensor during the helicopter outdoor free flight experiments are presented.

\section{Biomimetic optic flow sensing applied to spacecraft landing}

2.1 Autonomous lunar landing strategy

Lunar landing trajectory has been divided into four different phases in (Frapard et al., 1996) (see Fig. 3):

1. De-orbit Phase,

2. Approach Phase,

3. Final Descent,

4. Free Fall.

In this research work, a solution to the autonomy problem of the approach phase defined from the high gate (500m above ground level (AGL)) to the low gate (10m AGL) is studied. High gate corresponds to the height from which the landing site becomes visible from the spacecraft vision system. Low gate corresponds to the height from which visual contact with the landing site is no longer available due to the dust raised by the thrusters. Initial parameters are a horizontal speed $V_{x_{0}}=150 \mathrm{~m} / \mathrm{s}$, a vertical speed $V_{z_{0}}=-50 \mathrm{~m} / \mathrm{s}$, a pitch angle $\theta_{0}=-60^{\circ}$, a ground height $h_{0}=500 \mathrm{~m}$ and a mass $m_{l d r_{0}}=10^{3} \mathrm{~kg}$ (see Fig. 3).

This reference trajectory (Valette et al., 2010) is very similar to the one in the Apollo test case scenario used in (Izzo and de Croon, 2011; Izzo et al., 2011).

Solution to the problem must meet particularly demanding terminal constraints at the low gate $\left(h_{f}=10 \mathrm{~m}\right)$ as follows:

$-0 \leq V_{x_{f}} \leq 1 \mathrm{~m} / \mathrm{s}$,

$-1 \leq V_{z_{f}} \leq 0 \mathrm{~m} / \mathrm{s}$.

Designing an optimal control strategy for lunar landing also requires considering the propellant consumption.

The main challenge is that the entire state vector is not available from the measurement as can be seen on Fig. 4. For instance, velocities and position are 


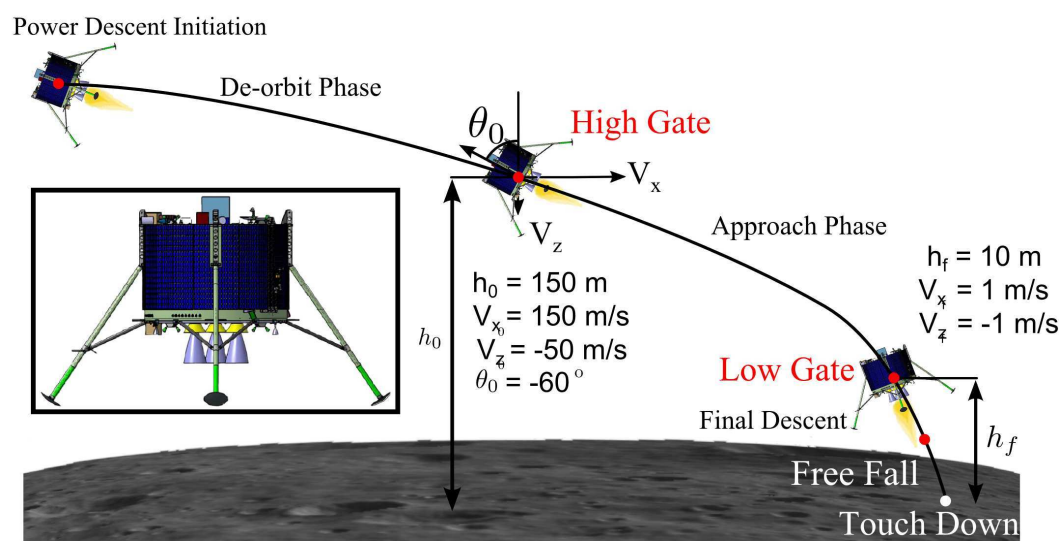

Fig. 3 Reference trajectory for lunar landing and 3D representation of the lunar module (courtesy: Astrium). The landing part addressed in this work is the approach phase defined between the high gate (500m AGL) and the low gate (10m AGL). The objectives of the lander is to reach the low gate $(10 \mathrm{~m}$ high) with both vertical and horizontal velocities lower than 1m/s. Figure modified from (Jean-Marius and Strandmoe, 1998; Valette et al., 2010).

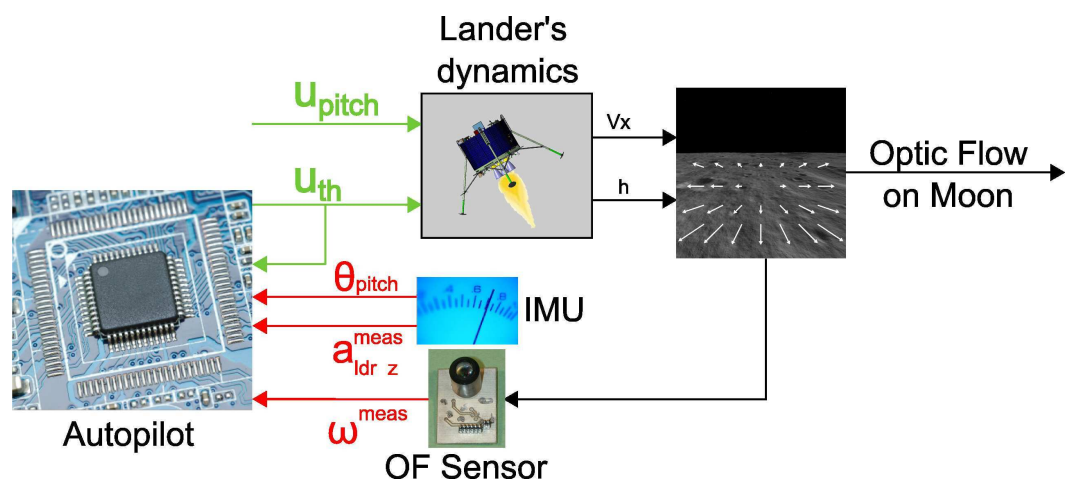

Fig. 4 Scheme of the closed loop system based on OF and pitch control. $\omega^{\text {meas }}$ the measured $\mathrm{OF}, u_{\text {pitch }}$ and $u_{t h}$ are the pitch angle and the main thruster control signal respectively. Concerning the measurements, the IMU assess the pitch angle $\theta_{\text {pitch }}$ and lander's accelerations both horizontal $\left(a_{l d r_{x}}\right)$ and vertical $\left(a_{l d r_{z}}\right)$. The mass $m_{l d r}$ is estimated from the sufficiently well known initial mass . Figure modified from (Valette et al., 2010).

neither measured nor estimated, only accelerations, angular position, mass and OF are measured and thus available to feed the controllers. To land safely on the moon the autopilot should be able to reduce the velocity vector magnitude and this is achieved by acting jointly on the lander's pitch and the lander's main thrust, the two available control signals. 
2.2 Lander's dynamic modeling and optic flow equations

The autopilot under consideration consists mainly of an OF-based control system operating in the vertical plane $(x, z)$, and controlling the spacecraft's mean thruster force and pitch angle. To stabilize the lander, it is necessary to cope with non-linearities and the inherent instability.

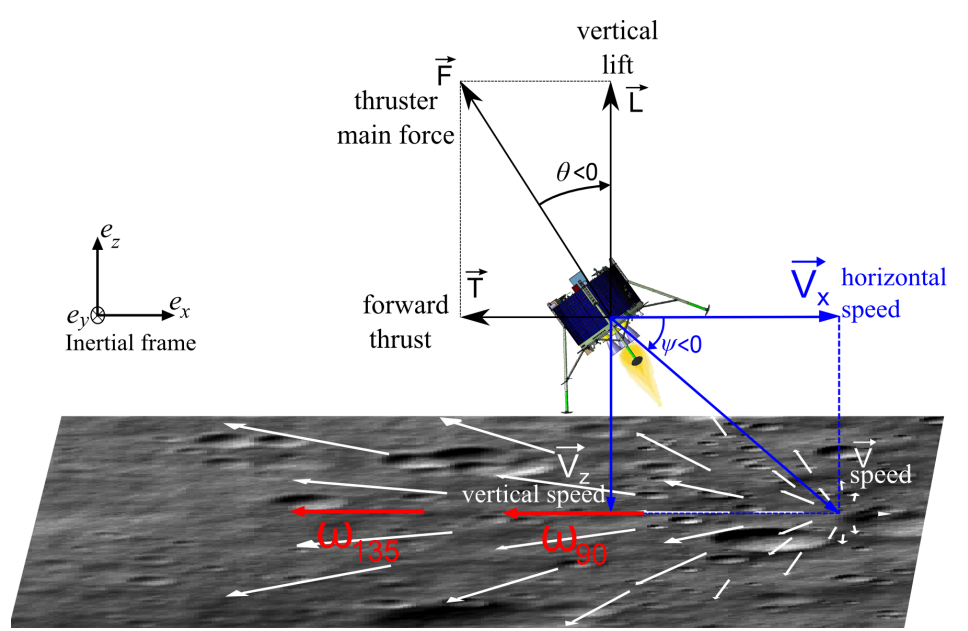

Fig. 5 Diagram of the lander, showing its speed vector $\mathbf{V}$, the mean thruster force $\mathbf{F}$ and its projections along the surge axis (X-axis) giving the forward thrust $\mathbf{T}$, and along the heave axis (Z-axis) giving the vertical lift $\mathbf{L}$.

Since there is no atmosphere on the Moon, the lander experiences neither wind nor drag. In the present model, heave and surge dynamics are coupled via the lander's pitch (see Fig. 5). It is worth noting that it is inappropriate to measure $\omega_{45^{\circ}}$ to determine the direction of the velocity vector, as the value $45^{\circ}$ remains close to the focus of expansion where the motion is always null (see Fig. 5).

The dynamic motion of the lander can be described in the time domain by the following dynamic system in the inertial frame associated with the vector basis $\left(\mathbf{e}_{\mathbf{x}}, \mathbf{e}_{\mathbf{y}}, \mathbf{e}_{\mathbf{z}}\right)$ :

$$
\left\{\begin{array}{l}
a_{l d r_{z}}(t)=\frac{\cos (\theta(t))}{m_{l d r}(t)} \cdot u_{t h}(t)-g_{\text {Moon }} \\
a_{l d r_{x}}(t)=\frac{\sin (\theta(t))}{m_{l d r}(t)} \cdot u_{t h}(t)
\end{array}\right.
$$

Where $u_{t h}=\|\mathbf{F}\|$ corresponds to the control force applied to the lander, $a_{l d r_{x, z}}$ are the lander's accelerations in the lunar inertial reference frame, $m_{l d r}$ stands for the lander's mass, $\theta$ is the pitch angle, $t$ denotes the time and $g_{M o o n}$ the lunar gravity constant $\left(g_{\text {Moon }}=1.63 \mathrm{~m} / \mathrm{s}^{2}\right)$. 
The lander's mass depends directly on the fuel consumption, as given by the following relation:

$$
\dot{m}_{l d r}=\frac{-1}{I_{s p} \cdot g_{\text {Earth }}} u_{t h}(t)
$$

where $I_{s p}=311 \mathrm{~s}$ corresponds to the specific impulse and $g_{\text {Earth }}=9.81 \mathrm{~m} / \mathrm{s}^{2}$ to the Earth's gravity. This means that:

$$
m_{l d r}(t)=m_{l d r}\left(t_{0}\right)-\frac{1}{I_{s p} . g_{E a r t h}} \int_{t_{0}}^{t} u_{t h}(\epsilon) d \epsilon
$$

where $m_{l d r}\left(t_{0}\right)=10^{3} \mathrm{~kg}$ is the lander's mass at high gate level.

Since the initial mass is known and the lander's mass depends linearly on the integral of the lander's thruster control signal, the mass can be computed and assessed at any time during the simulated descent.

The inner pitch control system is modeled as follows:

$$
\frac{I}{R} \frac{d^{2} \theta}{d t^{2}}=u_{p i t c h}(t)
$$

$u_{\text {pitch }}$ is the control input signal giving the spacecraft's pitch and $\theta$ is measured via an Inertial Measurement Unit, $I$ the moment of inertia of the lander and $R$ its radius.

Once the dynamic model of the spacecraft is defined, one needs to state the OF equations to find what information can be deduced from this visual cue.

The ground-truth OF $\omega_{g r d-t r h}$ can be described as the sum of the two distinct components defined by (Koenderink and van Doorn, 1987), i.e. the translational and rotational OF:

$$
\omega_{\text {grd }-t r h}=\omega_{T}+\omega_{R}
$$

The translational OF $\omega_{T}$ depends on the linear velocity $V$ expressed in the inertial frame, the distance from the ground $D$ in the gaze direction and the elevation angle $\Phi$ (i.e., the angle between the gaze direction and the heading direction).

$$
\omega_{T}=\frac{V}{D} \cdot \sin (\Phi)
$$

The rotational OF $\omega_{R}$ depends only on the vehicle's angular speed $\Omega_{j}$ expressed in the body fixed frame, where $j$ denotes the axis of rotation, and on the elevation angle $\lambda$ between the gaze direction and the axis of rotation.

$$
\omega_{R}=\Omega_{j} \cdot \sin (\lambda)
$$

Finally the general equation of the OF in the vertical plane is as follows:

$$
\omega_{g r d-t r h}=\frac{V}{D} \cdot \sin (\Phi)+\Omega_{j} \cdot \sin (\lambda)
$$


Under the assumption that the sensors are embedded on a gimballed system one can derive the expression of the OF measured in the vertical direction considering a pure translational motion $\left(\Omega_{j}=0\right)$. From Eq. (8), under the assumption of a practically flat ground (i.e. $D=h / \cos \left(\frac{\pi}{2}-\Phi+\Psi\right)$, where $\Phi-\Psi$ denotes the angle between the gaze direction and the local horizontal), and gimballed mounted sensors the ventral optic flow is defined as follows:

$$
\omega_{90}=\frac{V x}{h}
$$

where $V=V_{x} / \cos (\Psi)$.

2.3 Optic flow-based regulator for spacecraft landing

From Eq. (1) and modeling the thruster's dynamics by a first order transfer function with $\tau_{\text {thruster }}=100 \mathrm{~ms}$ such that $\tau_{\text {thruster }} \dot{u}_{t h}+u_{t h}=m_{l d r} \cdot u$, the Laplace transform of the heave dynamics $Z(s)$ can be written as follows:

$$
Z(s)=\frac{1}{s^{2}} \cdot\left[\left(\frac{1 / \tau_{\text {thruster }}}{1 / \tau_{\text {thruster }}+s} \cdot \cos (\theta) \cdot U(s)\right)-g_{\text {Moon }}\right]
$$

Where $U(s)$ is the Laplace transform of the control input signalu $(t)$.

The transfer function for the surge dynamics $G_{x}(s)$ can be written as follows:

$$
G_{x}(s)=\frac{X(s)}{U(s)}=\frac{1}{s^{2}} \cdot\left(\frac{1 / \tau_{\text {thruster }}}{1 / \tau_{\text {thruster }}+s} \cdot \sin (\theta)\right)
$$

For the lander model, if $a_{t h_{z}}$ is the vertical thruster's acceleration, we choose the following state vector $X=\left[\begin{array}{c}h \\ V_{z} \\ a_{t h_{z}}\end{array}\right]$ and the defined control inuput signal $u$. According to Eqs. (10) and (11), one can write:

$$
\left\{\begin{array}{c}
\dot{a}_{t h_{z}}=\frac{1}{\tau_{\text {thruster }}} \cdot\left[u-a_{t h_{z}}\right] \\
\dot{V}_{z}=a_{t h_{z}}-g_{M o o n} \\
\dot{h}=V_{z}
\end{array}\right.
$$

One can deduce from the Eq. (12), the state space representation:

$$
\dot{X}=A_{p} \cdot X+B_{p} \cdot u-g_{M o o n}
$$




$$
\begin{aligned}
{\left[\begin{array}{c}
\dot{h} \\
\dot{V_{z}} \\
a_{t h_{z}}
\end{array}\right] } & =\left[\begin{array}{ccc}
0 & 1 & 0 \\
0 & 0 & 1 \\
0 & 0 & \frac{-1}{\tau_{\text {thruster }}}
\end{array}\right] \cdot\left[\begin{array}{c}
h \\
V_{z} \\
a_{t h_{z}}
\end{array}\right] \\
+ & {\left[\begin{array}{c}
0 \\
0 \\
\frac{1}{\tau_{\text {thruster }}}
\end{array}\right] \cdot u-\left[\begin{array}{c}
0 \\
g_{M o o n} \\
0
\end{array}\right] }
\end{aligned}
$$

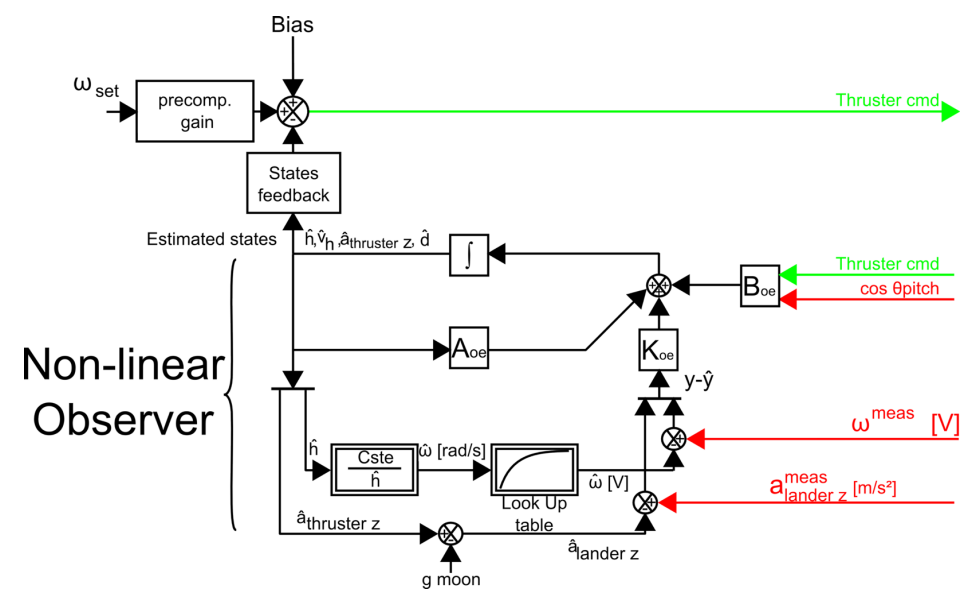

Fig. 6 The present autopilot makes use of a non-linear observer to provide the feedback control scheme with the state estimates. The observer was nonlinear since the ventral OF $\omega$ is by definition an inverse function of the controlled variable $h$. The state estimate of the $\mathrm{OF} \hat{\omega}$ in $\mathrm{rad} / \mathrm{s}$ was computed in terms of the ratio between a constant (the ground speed at a given working point) and the estimated height of the lander $\hat{h}$. The OF was then obtained via a look-up table. The measured acceleration of the lander $a_{\text {lander }}^{\text {meas }}$ served only to improve the state estimation. The estimated acceleration $\widehat{a}_{\text {lander }}$ was obtained by subtracting the approximate gravity on the Moon $g_{\text {moon }}$ from the estimated acceleration induced by the thruster $\widehat{a}_{\text {thruster }_{z}}$. Figure from (Valette et al., 2010).

The present spacecraft was modeled taking into account the thruster dynamics and a pure double integration between the acceleration and the altitude, using the state-space approach. The autopilot, which operated on the basis of a single OF measurement (that of the ventral OF), consisted of a visuomotor feedback loop driving the main thruster force. Since the vertical lift and the forward thrust are coupled, the loop controls both the heave and surge axes. The pitch angle $\theta$ was controlled by an external system which made the lander gradually pitch backwards from $-60^{\circ}$ to $-30^{\circ}$ throughout the landing approach. The autopilot presented in Fig. 6 was composed of (i) a precompensation gain, (ii) a non linear state observer, and (iii) a state feedback gain. The nonlinear state observer estimated the state vector $X$ on the basis of the ventral measured $\mathrm{OF} \omega^{\text {meas }}$, the lander acceleration, $a_{\text {lander }_{z}}$ and the lander 
pitch, $\theta$. The complete regulator combined the estimated states with the full state feedback control loop.

\subsubsection{State feedback control law design}

The autopilot kept the ventral OF of the simulated spacecraft at the set point $\omega_{\text {set }}$. This set point was compared with the product of the estimated state vector $\widehat{X}$ (see Fig. 6) and the state feedback gain $L_{s f}$ to generate the thruster command. The state feedback gain was calculated using the minimization criterion in the Linear Quadratic Regulator (LQR) method, using the following matrix: $A_{s f}=A_{p}, B_{s f}=B_{p}$ and $C_{s f}=\left[\begin{array}{lll}K_{\text {lin }} & 0 & 0\end{array}\right]$ and the state-cost ma$\operatorname{trix} Q_{c}=\left[\begin{array}{ccc}7.8 \cdot 10^{-4} & 0 & 0 \\ 0 & 0 & 0 \\ 0 & 0 & 0\end{array}\right]$ and $R_{c}=[1]$. To compute the $C_{s f}$ matrix, we linearized the expression for the OF near a set point. Here the set point was $h_{\text {lin }}=200 \mathrm{~m}, V_{x_{\text {lin }}}=50 \mathrm{~m} / \mathrm{s}$ and $\omega=14.3^{\circ} / \mathrm{s}$. The OF was defined as an inverse function of $h$. We therefore used the slope of the tangent to linearize the expression as follows:

$$
K_{l i n}=V_{x_{l i n}} \cdot \frac{d}{d h}\left(\frac{1}{h}\right)_{h=h_{l i n}}=\frac{-V_{x_{l i n}}}{h_{l i n}^{2}}
$$

\subsubsection{Nonlinear state observer design}

Since the system is observable, a state observer for $\widehat{\dot{X}}$ can be formulated as follows:

$$
\left\{\begin{array}{c}
\dot{\widehat{X}}=A_{o} \cdot \widehat{X}+B_{o} \cdot u+K_{o} \cdot(y-\widehat{y}) \\
\widehat{y}=C_{o} \cdot \widehat{X}+D_{o} \cdot u
\end{array}\right.
$$

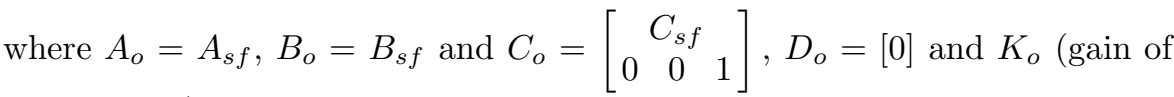
the observer) was also computed with LQR method, using $A_{o}$ and $C_{o}$ matrices. As shown in Fig. 6, the observer requires the value of the lander's acceleration $a_{l d r_{z}}$.

To achieve an integral control, the augmented state vector $X_{e}$ was thus defined: $X_{e}=\left[\begin{array}{c}X \\ d\end{array}\right]$ where $d$ stands for the disturbance. The new state matrices could therefore be written as follows:

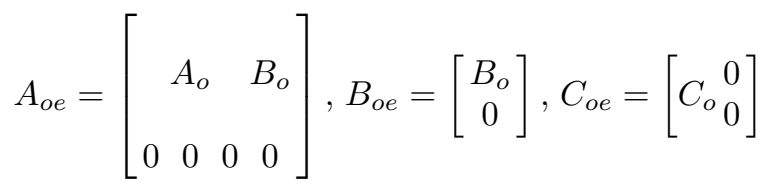

The state feedback gain $L_{s f e}$ was equal to $L_{s f e}=\left[L_{s f} 1\right]$. The observer gain was computed in the case of the extended state using the same method, 
with the new state matrix $\left(A_{o e}\right.$ and $\left.C_{o e}\right)$. The acceleration of the lander $\hat{a}_{l d r_{z}}$ was estimated by subtracting the lunar gravity $g_{M o o n}$ from the estimated engine thrust $\hat{a}_{t h_{z}}$ as shown on Fig. 6 .

The observer is initialized using a rough estimation of the initial height and vertical speed at high gate. The observer tolerates an uncertainty of about $20 \%$ in the estimation of the height and vertical speed.

\subsubsection{Autonomous landing simulation using the PANGU software program}
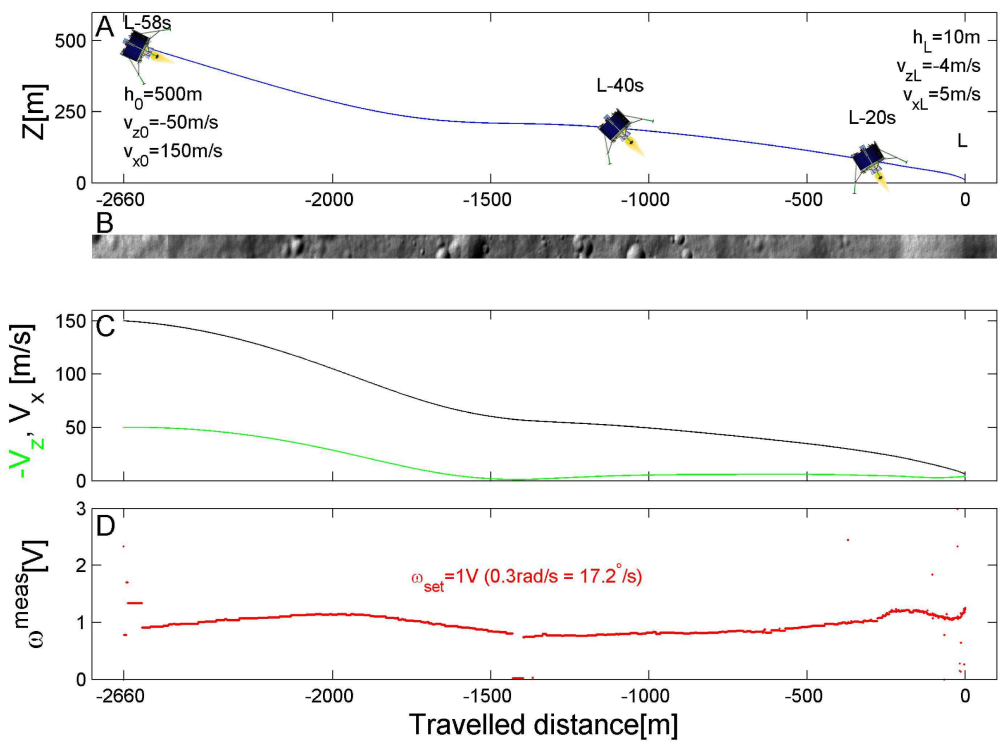

Fig. 7 Automatic landing based on a biomimetic OF sensor combined with a bio-inspired strategy. The OF sensor rests on a single pair of photoreceptors (i.e. two pixels), the mean orientation of which was maintained downwards. Figure from (Valette et al., 2010).

To ensure a soft landing, the lander had to reach a distance of approximately 10 meters from the ground (i.e., the low gate) at a residual velocity of one meter per second in both the horizontal and vertical directions. In (Valette et al., 2010), thanks to the biomimetic autopilot, the lander reached the low gate with greatly reduced horizontal and vertical speeds approximately equal to the required values as shown in Fig. 7. The lunar surface perceived by the lander consisted of gray-scale images generated by PANGU. In the presented simulation, an initial altitude $h_{0}=500 \mathrm{~m}$, an initial ground speed $V_{x_{0}}=150 \mathrm{~m} / \mathrm{s}$ and an initial vertical speed $V_{z_{0}}=-50 \mathrm{~m} / \mathrm{s}$ were adopted. The pitch angle $\theta$ was made to decrease exponentially from $-60^{\circ}$ to $-30^{\circ}$. As a consequence, the forward speed decreased quasi-exponentially as well (see Fig. $7 \mathrm{C}$ ), and so did the vertical speed (see Fig. 7C) since its integral $h$ was reduced 
quasi-exponentially to hold the measured OF $\omega_{\text {meas }}=\frac{V_{x}}{h}$ around the OF set point value $\omega_{\text {set }}$ (see Fig. 7D).

The spacecraft's simulated approach took $58.4 \mathrm{~s}$, which corresponds to the time required to reach the low gate. The lander reached the low gate at a final ground speed $V_{x_{f}}=5 \mathrm{~m} / \mathrm{s}$ and a final vertical speed $V_{z_{f}}=-4 \mathrm{~m} / \mathrm{s}$; the distance traveled by the lander during the landing was 2660 meters. The final horizontal and vertical speeds are slightly higher than required to meet strictly satisfy the speeds' criterion at low gate $(1 \mathrm{~m} / \mathrm{s})$.

In (Valette et al., 2010), the OF regulation principle was applied to autonomous lunar landing problems using an OF based feedback loop and tested by performing PANGU software simulations. The first simulations presented involved neuromorphic principles applied to monitoring and processing the 1-D OF on an autonomous visual-based extraterrestrial landing scenario. The autopilot generated the landing approach while monitoring only the ventral OF without the need to measure or estimate speed and height, and hence without the need for any bulky and power-consuming sensors. The main drawback with using the ventral OF exclusively is that the vertical dynamics of the lander is not taken into account with the consequence that the low gate cannot be reached with sufficiently low speeds as seen on Fig. 7. In the following, further OF measurements are introduced with an aim to recover the dynamics of the lander.

2.4 OF measurements $\omega_{90}$ and $\omega_{135}$ for velocity orientation estimation and control

To achieve a fully autonomous lunar landing, a possible way to improve the autopilot presented here would be to design a visual-based pitch control law to replace the fixed reference from $-60^{\circ}$ to $-30^{\circ}$ used in the current simulations.

In (Izzo et al., 2011; Valette et al., 2010), authors have shown the substance of the pitch control law in the design to achieve optimal performances since the system is underactuated

In the ongoing work, the main idea is to design an autopilot that keeps the main thrust antiparallel to the velocity vector orientation, in order to reduce the lander's fuel consumption as much as possible.

This principle defines pitch angle reference $\theta_{\text {ref }}$ which is fed into the pitch controller:

$$
\theta_{\text {ref }}=-\Psi-\frac{\pi}{2}
$$

Where $\Psi$ denotes the angle between the orientation of the speed vector and the local horizontal.

That strategy implies that in order to be able to strongly reduce the lander's speed during the approach phase, one needs to measure or estimate the velocity vector orientation $\Psi$ which is not an easy tasks considering the lack of appropriate sensors onboard the lander. The main question is how to fuse different visual angular speed measurements, to obtain useful information about 
unavailable measurement of the state vector. It is straightforward to note that the $\mathrm{OF}$ cue is related to the orientation of the velocity vector. One can derive the orientation angle $\Psi$ from OF sensors positioned in different directions by fusing $\omega_{90}=\frac{V x}{h}(9)$ and $\omega_{135}$ :

$$
\omega_{135}=\frac{V}{h / \cos (\pi / 4)} \cdot \frac{\sqrt{2}}{2}(\cos (\Psi)-\sin (\Psi))=\frac{\omega_{90}}{2} \cdot(1-\tan (\Psi))
$$

Finally one can obtain:

$$
\tan (\Psi)=1-2 \frac{\omega_{135}}{\omega_{90}}
$$

It is worth noting that both the horizontal and vertical dynamics are expressed within $\tan (\Psi)$ with $\tan (\Psi)=\frac{V z}{V x}$.

Thanks to Eq. (20) featuring only visual information, a pitch controller based on OF and pitch measurement $\theta$ provided by the IMU could be designed through Eq. (4) in order to ensure the colinearity between the lander's main thruster force and its velocity vector orientation.

The low speed visual motion sensors are thus the cornerstones of this autonomous lunar landing strategy. Since both the OF controller and the pitch controller are based on the output signals of OF sensors, it seemed to be worth testing the reliability of such sensors in real-life conditions. This is the aim of the following section.

\section{VMS-based OF measurements obtained onboard ReSSAC}

The low speed visual motion sensors used to measure the OF are strongly linked to the control signals of the dynamic system. This is why we developed and tested a new VMS dedicated to low angular speed measurements.

\subsection{Bio-inspired optic flow processing}

A mandatory step in the maturation of a technology is to design and embed the previously simulated device on a real-life complex system. In order to validate the feasibility of the theoretical work using the low speed VMS presented in Sec. ??, an experimental approach is presented.

A low resolution visual motion sensor based on a 6-pixels array and dedicated to a low speed range has been developed to demonstrate on Earth the feasibility of measuring the 1-D local angular speed on a lunar landing like scenario. We tested the sensor onboard an unmanned helicopter to validate the bio-inspired algorithm at relatively low ground speeds and relatively high ground heights and in the presence of strong natural disturbances (i.e. craft vibrations, uncontrolled illuminance, rough terrain, etc.).

This sensor is an updated version of the 2-pixel Local Motion Sensors designed by the biorobotic team on the basis of neurophysiological findings in flies (Franceschini et al., 1989). 


\subsection{Presentation of the low-speed visual motion sensor}

The new low-speed visual motion sensor (VMS) consists mainly of a low-cost plastic lens (CAX183 from Thorlabs, focal length 18.33mm, f-number 4.07) placed in front of an off-the-shelf photodiode array LSC from iC-Haus. The latter features six photodiodes, each of which has a large sensitive area $(300 \times$ $1600 \mu \mathrm{m}$ ) and an integrated preamplifier. The LSC conveys the six photodiode signals to a hybrid analog/digital processing algorithm which computes the OF value $\omega_{\text {meas }}$. A custom-made protective case was added to protect the lowweight sensor and the optical assembly from unfavorable weather conditions. $29.4 \mathrm{~g}$.

The new visual motion sensor and its custom-made protective case weighed

Many of the parameters of the original visual motion detecting scheme presented in (Blanes, 1986; Pichon et al., 1989) have been updated, especially in terms of interreceptor angles and cut-off frequencies of the temporal filters.

The six optical axes formed by the photodiodes are separated by an angle called the interreceptor angle $\Delta \varphi$. By defocusing the lens (i.e., by adjusting the distance between the lens and the photosensors), we obtained a Gaussian angular sensitivity functions for each photoreceptor with a correlation coefficient greater than $99 \%\left(R_{L S C}^{2}>0.990\right)$, These features were assessed by slowly rotating the lens in front of a point light source placed at a distance of $85 \mathrm{~cm}$. The local 1-D angular speed $\omega_{\text {meas }}$ measured by the sensor was defined as the ratio between the interreceptor angle $\Delta \varphi$ and the time elapsing $\Delta t$ between the moments when two adjacent photodiode signals reached the threshold. $\Delta t$ represents the "time of travel" of a any given contrast feature passing from the optical axis of one photodiode to the optical axis of the neighboring one.

$$
\omega_{\text {meas }}=\frac{\Delta \varphi}{\Delta t}
$$

In (Expert et al., 2011), the measurement range of the sensor covered a large range of high speeds from $50^{\circ} / \mathrm{s}$ to $300^{\circ} / \mathrm{s}$, whereas the present study focused on low velocities giving a range of $1.5^{\circ} / \mathrm{s}$ to $25^{\circ} / \mathrm{s}$, which is more than tenfold slower. In order to stay in the same range of $\Delta t$, whose accuracy of measurement depends on the microcontroller's sampling frequency, we therefore had to narrow $\Delta \varphi$.

The large $18.33 \mathrm{~mm}$ focal length increases the defocalizing effects of the lens, giving a suitably small mean interreceptor angle of $\overline{\Delta \varphi}=1.4^{\circ}$. The second advantage of the defocusing process is that it adds a blurring effect giving each pixel a Gaussian-shaped angular sensitivity function with a half width of similar size $\Delta \rho=1.4^{\circ}$. The resolution attained here is very similar to that measured in the common housefly compound eye (Kirschfeld and Franceschini, 1968).

$$
\Delta \varphi=\Delta \rho
$$

The acceptance angle, defined by $\Delta \rho$, acts as an optical low pass spatial filter. Achieving $\Delta \rho / \Delta \varphi$ ratio of 1 made it possible for the OF sensor to respond to 
contrasting features of high spatial frequency.

With $\Delta \varphi=\Delta \rho=1.4^{\circ}$, the overall FOV of the VMS was $10.28^{\circ}$.

The general processing algorithm underlaying the VMS consists of two parts: an analog processing part that converts the six photodiode signals into electrical signals with a high signal-to-noise ratio, and a digital processing part that simultaneously computes five OF values plus the OF median value (see Fig. 8). The analog processing part begins with a programmable gain connected to the microcontroller via a SPI communication bus (Ruffier and Expert, 2012). A bandpass filter then differentiates the visual signal and acts as an anti-aliasing filter.

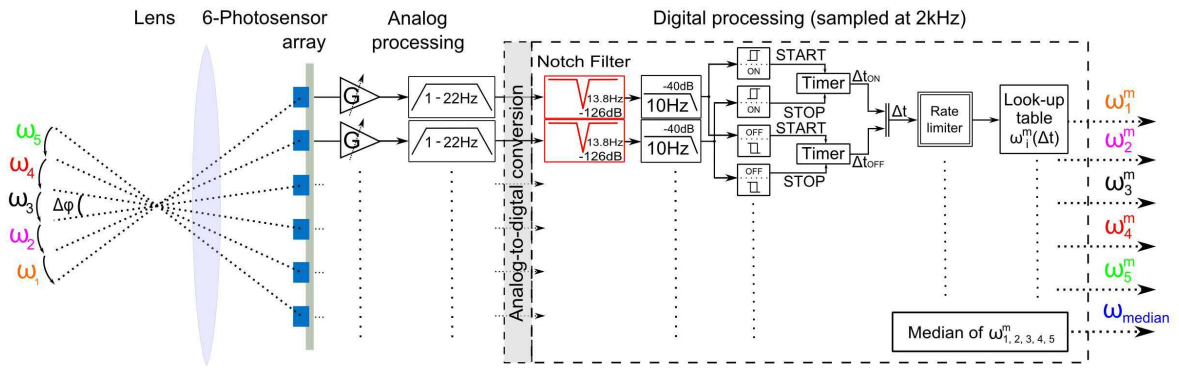

Fig. 8 General processing architecture of the low-speed visual motion sensor. First of all, the spatial sampling and low-pass filtering steps are carried out by the defocused lens. The six photodiode signals are amplified with programmable gain to increase the signal to noise ratio, before being filtered by an analog bandpass filter $(1-22 \mathrm{~Hz})$. The digital stage begins with a second order fixed-point notch filter centered on the helicopter's main rotor frequency $(13.8 \mathrm{~Hz})$. It is followed by a second order fixed-point low pass (cut-off frequency $=10 \mathrm{~Hz}$ ). A hysteresis thresholding process is associated with the computation of the time $\Delta t$ elapsing between two adjacent signals (of similar ON or OFF contrast polarity). Lastly, after an outlier filtering step, the output signal of the 1-D visual motion sensor is obtained from a precomputed look-up table and the median value is calculated. Figure modified from (Roubieu et al., 2011).

The digital processing algorithm starts with a second order fixed-point notch filter (rejection factor $Q=6.9$ ) whose center frequency was tuned to the helicopter's main rotor frequency $(13.5 \mathrm{~Hz})$. Its transfer function has been defined as follows (Orfanidis, 1995):

$$
H_{n o t c h}(z)=b \frac{1-2 \cos \left(\omega_{0}\right) z^{-1}+z^{-2}}{1-2 b \cos \left(\omega_{0}\right) z^{-1}+(2 b-1) z^{-2}}
$$

with

$$
b=\frac{1}{1+\frac{\sqrt{1-G_{B}^{2}}}{G_{B}} \tan \left(\frac{\Delta \omega}{2}\right)}
$$

where $\Delta \omega$ is the full width at a level $G_{B}^{2}$ and $\omega_{0}$ is the center frequency. We chose $\omega_{0}=2 \cdot \pi \frac{f_{s}}{f_{0}}, \Delta \omega=2 \cdot \pi \frac{\Delta f}{f_{s}}$ with $\Delta f=2 H z$ and $G_{B}^{2}=-3 d B$. 
As the visual angular speed $\omega_{g r d-t r h}$ is quite low, the temporal frequency $f_{t}$ of the visual signals derived from the features of the environments is also quite low, as expressed by the following equation (Landolt and Mitros, 2001):

$$
f_{t}=\omega_{g r d-t r h} \cdot f_{\text {spatial }}
$$

where $f_{\text {spatial }}$ is the spatial frequency associated with the contrasting pattern.

Therefore, a second order fixed-point low pass filter was used to enhance the signal-to-noise ratio by removing the noise remaining at frequencies higher than $10 \mathrm{~Hz}$.

The OF algorithm ("Time of travel scheme") implemented here consists mainly of a hysteresis thresholding process with separate ON and OFF pathways (Blanes, 1986; Pichon et al., 1989; Viollet and Franceschini, 1999b; Ruffier et al., 2003; Roubieu et al., 2011) followed by the $\Delta t$ computation, the result of which is fed into a correspondance lookup table. Lastly, the five simultaneously computed OFs $\omega_{i}^{m}$ are fused by a median operator to increase the refresh rate robustness of the output (Roubieu et al., 2011).

The microcontroller used for this purpose (dsPIC33FJ128GP802) operates at a sampling frequency of $2 k H z$, except for the digital filters, which are sampled at a rate of $500 \mathrm{~Hz}$. Special efforts were made to optimize the algorithm, and a computational load of only $17 \%$ was eventually obtained.

\subsection{Characterization of the Visual Motion Sensor (VMS)}

The characteristics of the present visual motion sensor (VMS) were assessed by performing OF measurements under controlled motion conditions (orientation and velocity) outdoors. Pure rotational motion was applied to the sensor at angular speeds ranging from $1^{\circ} / \mathrm{s}$ to $20^{\circ} / \mathrm{s}$ using a previously described outdoor set-up (Expert et al., 2011). The triangular response pattern obtained corresponds closely to the reference angular speed (see Fig. 9). It can therefore be said that this new tiny sensor is able to accurately compute the 1-D visual angular speed during a rotational motion within its operating range. The refresh rate is defined as the ratio between the total number of measurements occurring within the acceptable range $\left[1.5^{\circ} / s-25^{\circ} / s\right]$ and the time elapsing. The mean refresh rate achieved during the dynamic performances evaluation was $f_{\text {refresh }}=6.6 \mathrm{~Hz}$ : this value depends on the richness of the visual environment, as well as on the actual angular speed.

\subsection{Free-ying results with the airborne visual motion sensor}

The VMS dynamic performance was then studied on a six-DOF UAV during free flight over fields. A Yamaha Rmax helicopter was used in the framework of the ONERA's ReSSAC project. The helicopter characteristics in terms of mass balance have been described in (Watanabe et al., 2010). Its mass (80kg), its 


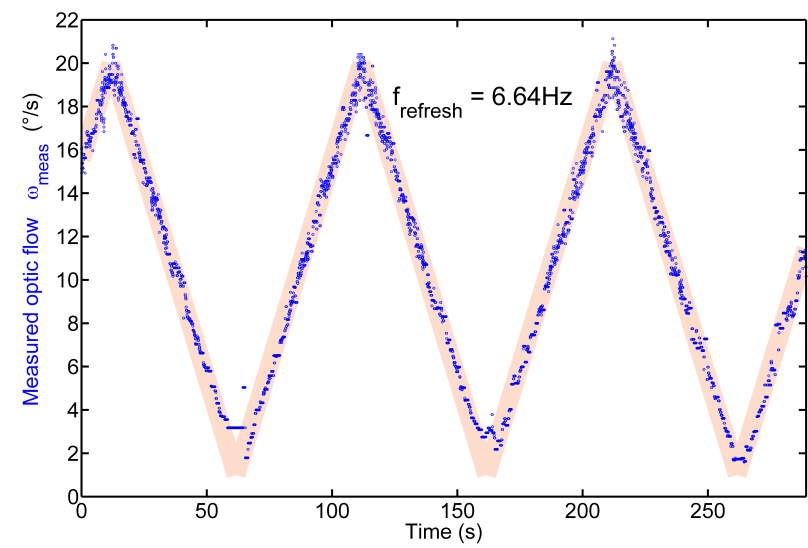

Fig. 9 Dynamic outdoor response of the low-speed VMS (blue), as compared with the ground-truth OF (red). The visual motion sensor was rotated by means of a conveyor belt driven by a stepping motor (103H5208-0440 from Sanyo-Denki) (Expert et al., 2011). Rotations from $1 \% \mathrm{~s}$ to $20^{\circ} / \mathrm{s}$ were applied to the sensor, which is designed to operate in the $1.5^{\circ} / \mathrm{s}$ to $25^{\circ} / \mathrm{s}$ range. The OF measured closely matched the reference signal, with a refresh rate of $6.64 \mathrm{~Hz}$. Since no synchronization signal was available, the ground-truth OF has been roughly synchronized here.

flight envelope and the vibration dynamics due to the main rotor's rotational speed presented us with quite a challenging ground-truth OF profile. The flight was performed in South-western France, mid-July at about 5pm on a bright sunny day: the mean illuminance was approximately $10000 \mathrm{~lx}$.

Onboard the ReSSAC helicopter, the 1-D local OF measured is subject to several variations as follows. Since the roll and pitch angles remain small throughout flight, the distance to the ground in the gaze direction $D$ can be approximated as $D \approx \frac{h}{\cos (\varphi) \cdot \cos (\theta)}$, where $\varphi$ denotes the roll angle, $\theta$ the pitch angle and $h$ the local ground height.

In our case, $\Phi=-\theta+\Psi+\frac{\pi}{2}$ (with the sensor oriented downward, $\Psi<0$, $\theta<0), \lambda=\frac{\pi}{2}$ and $\Omega_{j}=\Omega_{2}$, where $\Omega_{2}$ is the pitch angular velocity defined in the body fixed reference frame, the ground-truth OF (see (8)) is therefore computed as follows:

$$
\omega_{g r d-t r h}=\left(\frac{V}{h} \cdot \cos (\theta) \cdot \cos (\varphi) \cdot \sin \left(\theta+\Psi+\frac{\pi}{2}\right)\right)+\Omega_{2}
$$

During the experiment described below, the ground-truth OF $\omega_{\text {grd-trh }}$ was computed using data from the IMU, the GPS (OEM4 G2 from NovAtel) and the data grabbed by a LIDAR (Sick LDMRS 400001) during previous GPS assisted flights over the same fields.

The low speed visual motion sensor was embedded in the front part of ReSSAC helicopter and pointed vertically downwards with a clear FOV.

Fig. 10 shows the response of the low-speed visual motion sensor mounted onboard the unmanned ReSSAC helicopter. Despite the complex ground-truth 

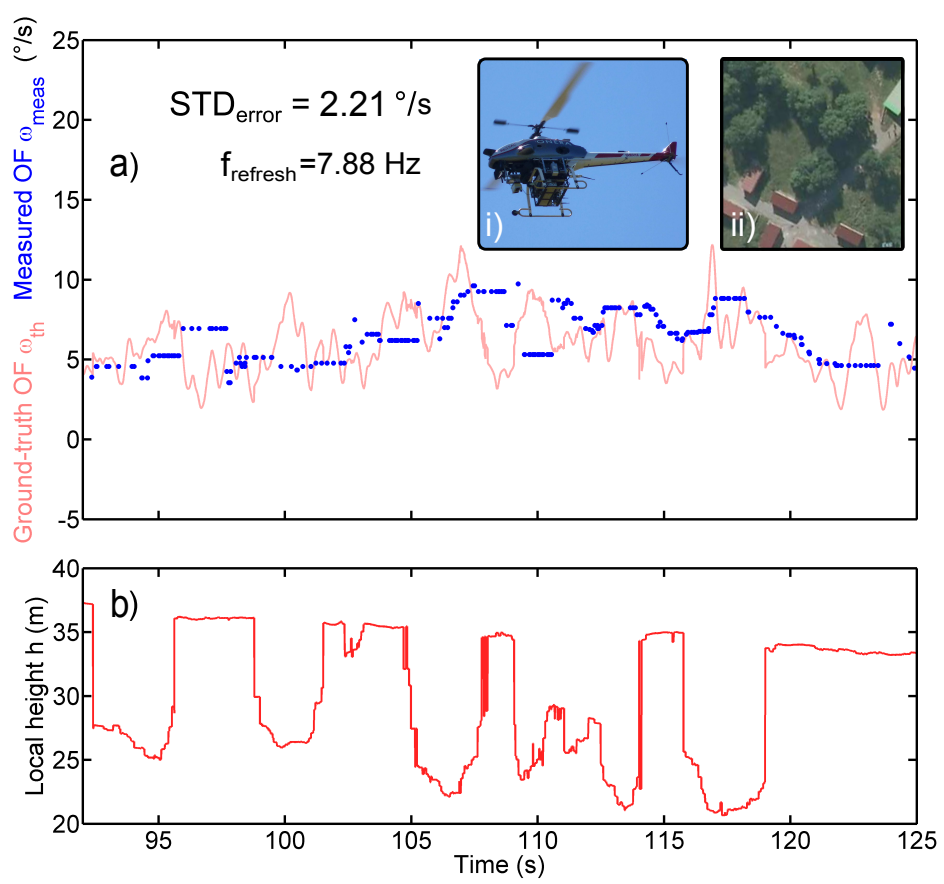

Fig. 10 Low-speed visual motion sensor and flight data sensed on-board the ReSSAC UAV. (a) Ground-truth OF (see (24)) (red) and measured OF $\omega_{\text {meas }}=\Delta \varphi / \Delta t$ (blue dots). Despite the strong variations mainly due to vibrations, the low-speed visual motion sensor's output closely matched the ground-truth $\mathrm{OF}$, giving a standard deviation of $2.21^{\circ} / \mathrm{s}$ and a refresh rate of $7.88 \mathrm{~Hz}$. The effects of strong variations in the local height due to the successive trees and houses are directly reflected in the low-speed VMS measurement signal. (i) ReSSAC unmanned helicopter in-flight (ii) Aerial view of the flight environment obtained on http://geoportail.fr. (b) Local ground height measured by combining GPS data and previously mapped LIDAR data. The nominal height was around $40 \mathrm{~m}$. But due to the variable relief (houses, etc.), the local groundheight often changed suddenly by 15 meters.

OF, the visual motion sensor responded appropriately to the visual stimuli. The standard deviation of the error between the ground-truth OF $\omega_{\text {grd-trh }}$ and the measured OF $\omega_{\text {meas }}$ was less than $2.25^{\circ} / \mathrm{s}$, which is quite low. The refresh rate $f_{\text {refresh }}$ was greater than $7.8 \mathrm{~Hz}$, which is even slightly higher than in the dynamic measurements performed during a rotating motion on ground. Fig. 10.b, giving the local ground height shows how well the sensor responded to its visual environment. Once again, the low-speed VMS accurately sensed these height variations and yielded similar values to the ground-truth value.

The robust and accurate performances observed during this experiment show that the low-speed visual motion sensor is highly suitable for use in many high-scaled robotic applications. 


\section{Conclusion}

In this chapter, we gathered several core technologies to achieve an autonomous landing approach based on low-speed optic flow (OF) sensors.

We presented preliminary results concerning the control scheme, along with the intended control strategy primarily based on the OF regulation scheme. We distinguish two parallel control loops, the one acting on the main thruster, the second one acting on the lander's pitch angle.

The aim is to control the lander's dynamics by exploiting OF measurements and keeping the main thruster force collinear to the velocity vector at all times. The challenge is to provide the lander with a near optimal descent trajectory in terms of its fuel consumption. The main benefit of this biologically inspired approach is that it avoids the estimation process of both height and velocity.

We showed that a sensor assembly based on two OF sensors oriented downwards in different directions $\left(90^{\circ}\right.$ and $135^{\circ}$ from the local horizontal) yields a direct estimate of the orientation $\Psi$ of the velocity vector. The latter can then be used as a reference signal for the pitch angle controller. The next step will be to develop full simulations featuring the control loops to attain at low gate $\left(h_{f}=10 \mathrm{~m}\right)$ the final velocity conditions stated in the reference scenario $\left(V_{x_{f}}=1 \mathrm{~m} / \mathrm{s}, V_{z_{f}}=-1 \mathrm{~m} / \mathrm{s}\right)$. The loop controlling the main thruster will be designed using OF cue. Secondly the gimballed setup used in simulations needs to be addressed. Since one of the main benefits of using OF cues is mass saving and simplicity, a gimballed system is not suited for this purpose. Increasing the number of VMS and thus enlarging the sensory FOV is a potential way to achieve such challenge.

After introducing the specific lunar landing approach, we presented a new lightweight visual motion sensor able to compute accurately the $\mathrm{OF}$ in the range experienced during the approach phase of a lunar landing. This new VMS has been developed, and then tested both on the ground and in flight onboard an unmanned helicopter flying over an unknown complex outdoor environment and under real-life dynamic and vibratory conditions. Encouraging results of this experiment showed that this sensor is perfectly suited for aeronautics or aerospace applications since it sensed accurately the local 1-D angular speed ranging from $1.5^{\circ} / \mathrm{s}$ to $25^{\circ} / \mathrm{s}$ with a quite frequently refreshed measurement.

\section{Acknowledgment}

We are most grateful to G. Graton, and F. Expert for their fruitful suggestions and comments during this study. We thank P. Mouyon, H. de Plinval, A. Amiez and V. Fuertes for the successful flight of ReSSAC helicopter, T. Rakotomamonjy and A. Piquereau for their expertise in helicopter dynamics, M. Boyron for his assistance with the electrical design, J. Diperi for his involvement in the mechanical design and J. Blanc for improving the English manuscript. 


\section{References}

Barrows, G. and Neely, C. (2000). Mixed-mode VLSI optic flow sensors for inflight control of a Micro Air Vehicle. In in SPIE : Critical technologies for the future of computing, volume 4109, pages 52-63, San Diego, CA, USA.

Beyeler, A., Zufferey, J., and Floreano, D. (2009). optipilot: control of take-off and landing using optic flow. In European Micro Aerial Vehicle Conference.

Blanes, C. (1986). Appareil visuel élementaire pour la navigation à vue d'un robot mobile autonome. Master's thesis, Master thesis in Neurosciences (DEA in French), Neurosciences, Univ. Aix-Marseille II, Advisor: N. Franceschini.

Blanes, C. (1991). Guidage visuel d'un robot mobile autonome d'inspiration bionique. Ph.D. thesis, INP Grenoble.

Braun, R. and Manning, R. (2006). Mars exploration entry, descent and landing challenges. In the proceedings of the IEEE Aerospace Conference, Big Sky, Montana. Pasadena, CA : Jet Propulsion Laboratory, National Aeronautics and Space Administration, 2006.

Cheng, Y. and Ansar, A. (2005). Landmark based position estimation for pinpoint landing on mars. In the proceedings of the IEEE International Conference on Robotics and Automation (ICRA), pages $1573-1578$.

Collett, T. S. and Land, M. F. (1975). Visual control of flight behaviour in the hoverfly Syritta pipiens. Journal of Comparative Physiology A: Neuroethology, Sensory, Neural, and Behavioral Physiology, 99(1), 1-66.

Conroy, J., Gremillion, G., Ranganathan, B., and Humbert, J. (2009). Implementation of wide-field integration of optic flow forautonomous quadrotor navigation. Autonomous Robots, 27, 189-198.

De Lafontaine, J. (1992). Autonomous spacecraft navigation and control for comet landing. Journal of Guidance, Control, and Dynamics, 15(3), 567576 .

Dubois-Matra, O., Parkes, S., and Dunstam, M. (2009). Testing and validation of planetary vision-based navigation systems with pangu. In the proceedings of the 21st International Symposium on Space Flight Dynamics (ISSFD), Toulouse, France.

Expert, F., Viollet, S., and Ruffier, F. (2011). Outdoor field performances of insect-based visual motion sensors. Journal of Field Robotics, 28 (4), 529-541.

Expert, F., Roubieu, F. L., and Ruffier, F. (2012). Interpolation based "time of travel" scheme in a visual motion sensor using a small $2 \mathrm{~d}$ retina. In the proceedings of the IEEE Sensors Conference, pages 2231-2234, Taipei, Taiwan.

Flandin, G., Polle, B., Frapard, B., Vidal, P., Philippe, C., and Voirin, T. (2009). Vision based navigation for planetary exploration. In the proceedings of the 32nd Annual AAS Rocky Mountain Guidance and Control Conference.

Franceschini, N. (1985). Early processing of colour and motion in a mosaic visual system. Neurosc Res Suppl, 2, 17-49. 
Franceschini, N. (1999). De la mouche au robot : reconstruire pour mieux comprendre, volume Cerveaux et machines. inconnu.

Franceschini, N. and Chagneux, R. (1997). Repetitive scanning in the fly compound eye. In Gttingen Neurobiology Report, volume 2. Thieme.

Franceschini, N., Riehle, A., and Nestour, A. L. (1989). Facets of Vision, chapter Directionally Selective Motion Detection by Insect Neurons, pages 360-390. Springer-Verlag.

Franceschini, N., Pichon, J. M., and Blanes, C. (1991). Real time visuomotor control: from flies to robots. In the proceedings of the IEEE Conference on Advanced Robotics (ICAR91), pages 931-935, Pisa, Italy.

Franceschini, N., Pichon, J. M., and Blanes, C. (1992). From insect vision to robot vision. Philosophical Transactions of the Royal Society B: Biological Sciences, 337 (1281), 283-294.

Franceschini, N., Pichon, J. M., and Blanes, C. (1997). Bionics of visuo-motor control. In T. Gomi, editor, Evolutionary Robotics: From Intelligent Robots to Artificial Life, pages 49-67. AAI Books, Ottawa.

Franceschini, N., Ruffier, F., and Serres, J. (2007). A bio-inspired flying robot sheds light on insect piloting abilities. Current Biology, 17(4), 329-335.

Franceschini, N., Ruffier, F., and Serres, J. (2009). Obstacle avoidance and speed control in insects and micro-aerial vehicles. Acta Futura, 3(4), 15-34.

Franceschini, N., Ruffier, F., and Serres, J. (2010). Encyclopedia of Aerospace Engineering, chapter Biomimetic Optic Flow Sensors and Autopilots for MAV Guidance, page Chapter E309.

Frapard, B., Champetier, C., Kemble, S., Parkinson, B., Strandmoe, S., and M., L. (1996). Vision-based gnc design for the leda mission. In the proceedings of the 3rd International ESA Conference on Spacecraft GNC, pages 411-421, Noordwijk, the Netherlands.

Frapard, B., Polle, B., Flandin, G., Bernard, P., Vétel, C., Sembely, X., and Mancuso, S. (2002). Navigation for planetary approach and landing. In the proceedings of the 5th International ESA Conference on Spacecraft GNC, Rome, Italy.

Garratt, M. and Chahl, J. (2008). Vision-based terrain following for an unmanned rotorcraft. Journal of Field Robotics, 25, 284-301.

Green, W., Oh, P., and Barrows, G. (2004). Flying insect inspired vision for autonomous aerial robot maneuvers in near-earth environments. In IEEE International Conference on Robotics and Automation (ICRA).

Griffiths, S., Saunders, J., Curtis, A., Barber, B., McLain, T., and Beard, R. (2006). Maximizing miniature aerial vehicles. Robotics \& Automation Magazine, IEEE, 13, 34-43.

Heisenberg, M. and Wolf, R. (1984). Vision in Drosophila. Springer-Verlag, New York.

Herisse, B., Hamel, T., Mahony, R., and Russotto, F.-X. (2012). Landing a vtol unmanned aerial vehicle on a moving platform using optical flow. IEEE transaction on robotics, 28(1), 77-89.

Hrabar, S., Sukhatme, G., Corke, P., Usher, K., and Roberts, J. (2005). Combined optic-flow and stereo-based navigation of urban canyons for a uav. 
In the proceedings of the International Conference on Intelligent Robots and Systems (IROS), pages 3309-3316.

Indiveri, G., Kramer, J., and Kocj, C. (1996). System implementations of analog vlsi velocity sensors. IEEE Micro, 16 (5), 40-49.

Izzo, D. and de Croon, G. (2011). Landing with time-to-contact and ventral optic flow estimates. Journal of Guidance, Control, and Dynamics, 35 (4), $1362-1367$.

Izzo, D., Weiss, N., and Seidl, T. (2011). Constant-optic-flow lunar landing: Optimality and guidance. Journal of Guidance, Control, and Dynamics, 34, 1383-1395.

Janschek, K., Tchernykh, V., and Beck, M. (2006). Performance analysis for visual planetary landing navigation using optical flow and dem matching. In the proceedings of the AIAA Guidance, Navigation and Control Conference and Exhibit.

Jean-Marius, T. and Strandmoe, S. E. (1998). Integrated vision and navigation for a planetary lander. Technical report, AEROSPATIAL, Espace et Défense, Les Mureaux-France. ESA, Estec.

Kawaguchi, J., Hashimoto, T., Misu, T., and Sawai, S. (1999). An autonomous optical guidance and navigation around asteroids. Acta Astronautica, 44(5), 267-280.

Kendoul, F., Nonami, K., Fantoni, I., and Lozano, R. (2009). An adaptive vision-based autopilot for mini flying machines guidance, navigation and control. Autonomous Robots, 27, 165-188.

Kerhuel, L., Viollet, S., and Franceschini, N. (2012). The vodka sensor: A bioinspired hyperacute optical position sensing device. IEEE Sensors Journal, 12(2), 315-324.

Kirschfeld, K. and Franceschini, N. (1968). Optische eigenschaften der ommatidien im komplexauge von Musca. Kybernetik, 5, 47-52.

Koenderink, J. and van Doorn, A. (1987). Facts on optic flow. Biological Cybernetics, 56, 247-254.

Landolt, O. and Mitros, A. (2001). Visual sensor with resolution enhancement by mechanical vibrations. Autonomous Robots, 11(3), 233-239.

Mahony, R., Corke, P., and Hamel, T. (2008). A dynamic image-based visual servo control using centroid and optic flow features. Journal of Dynamic Systems, Measurement, and Control, 130(1), 1-12.

Moeckel, R. and Liu, S.-C. (2007). Motion detection circuits for a time-totravel algorithm. IEEE International Symposium on Circuits and Systems. (ISCAS), New orleans, LA, USA, 3079-3082.

Mourikis, A. I., Trawny, N., Roumeliotis, S. I., Johnson, A. E., Ansar, A., and Matthies, L. (2009). Vision-aided inertial navigation for spacecraft entry, descent, and landing. IEEE Transactions on Robotics, 25(2), 264-280.

Netter, T. and Franceschini, N. (2002). A robotic aircraft that follows terrain using a neuromorphic eye. Intelligent Robots and Systems, 2008. IROS 2008. IEEE/RSJ International Conference on, 1, 129-134.

Orfanidis, S. (1995). Introduction to signal processing. Prentice-Hall, Inc. Upper Saddle River, NJ, USA. 
Parkes, S., Dunstan, M., Matthews, D., Martin, I., and Silva, V. (2003). Lidarbased gnc for planetary landing: Simulation with pangu. In the proceedings of the DASIA (Data Systems in Aerospace), pages 18.1-18.12.

Parkes, S., Martin, I., Dunstan, M., and Matthews, D. (2004). Planet surface simulation with pangu. In the proceedings of the 8th International Conference on Space Operations (SpaceOps).

Parkes, S. M. and Silva, V. (2002). Gnc sensors for planetary landers : a review. In the proceedings of the DASIA (Data Systems in Aerospace), pages 1-9.

Pichon, J., Blanes, C., and Franceschini, N. (1989). Visual guidance of a mobile robot equipped with a network of self-motion sensors. Mobile robots $I V$, SPI Volume 1195, 44-53.

Roubieu, F., Expert, F., Boyron, M., Fuschlock, B., Viollet, S., and Ruffier, F. (2011). A novel 1-gram insect based device measuring visual motion along 5 optical directions. In the proceedings of the IEEE Sensors conference, pages 687-690, Limerick, Ireland.

Roubieu, F. L., Serres, J., Franceschini, N., Ruffier, F., and Viollet, S. (2012). A fully-autonomous hovercraft inspired by bees; wall-following and speed control in straight and tapered corridors. In IEEE International Conference on Robotics and Biomimetics (ROBIO), Guangzhou, China.

Roumeliotis, S., Johnson, A., and Montgomery, J. (2002). Augmenting inertial navigation with image-based motion estimation. In the proceedings of the IEEE International Conference on Robotics and Automation (ICRA), volume 4, pages 4326-4333.

Ruffier, F. (2004). PILOTE AUTOMATIQUE BIOMIMETIQUE Système générique inspiré du contrôle visuomoteur des insectes pour : le décollage, le suivi de terrain, la réaction au vent et látterrissage automatiques dún micro-aéronef. Ph.D. thesis, INP Grenoble.

Ruffier, F. and Expert, F. (2012). Visual motion sensing onboard a 50-g helicopter flying freely under complex VICON-lighting conditions. In the proceedings of the International Conference on Complex Medical Engineering, pages 634-639, Kobe, Japan.

Ruffier, F. and Franceschini, N. (2003). Octave, a bioinspired visuo-motor control system for the guidance of micro-air vehicles. In A. Rodriguez-Vazquez, D. Abbott, and R. Carmona, editors, the proceedings of the Conference on Bioengineered and Bioinspired Systems, SPIE, volume 5119, pages 1-12, Maspalomas, Spain. Bellingham, USA.

Ruffier, F. and Franceschini, N. (2004). Visually guided micro-aerial vehicle: automatic take off, terrain following, landing and wind reaction. In the proceedings of the IEEE International Conference on Robotics and Automation (ICRA 2004), Coimbra, Portugal.

Ruffier, F. and Franceschini, N. (2005). Optic flow regulation: the key to aircraft automatic guidance. Robotics and Autonomous Systems, 50, 177 194.

Ruffier, F., Viollet, S., Amic, S., and Franceschini, N. (2003). Bio-inspired optical flow circuits for the visual guidance of micro-air vehicles. In the proceedings of the IEEE International Symposium on Circuits and Systems 
(ISCAS), volume 3, pages 846-849, Bangkok, Thailand.

Schilstra and Hateren (1999). Blowfly flight and optic flow. 1. thorax kinematics and flight dynamics. J Exp Biol, 202 (Pt 11), 1481-1490.

Shang, Y. and Palmer, P. (2009). The dynamic motion estimation of a lunar lander. In the proceedings of the 21st ISSFD, Toulouse, France.

Strandmoe, S., Jean-Marius, T., and Trinh, S. (1999). Toward a vision based autonomous planetary lander. In $A I A A$, pages AIAA-99-4154.

Tammero, L. F. and Dickinson, M. H. (2002). The influence of visual landscape on the free flight behavior of the fruit fly drosophila melanogaster. Journal of Experimental Biology, 205, 327-343.

Tchernykh, V., Beck, M., and Janschek, K. (2006). An embedded optical flow processor for visual navigation using optical correlator technology. In the proceedings of the IEEE/RSJ International Conference on Intelligent Robots and Systems, pages 67-72, Beijing.

Trawny, N., Mourikis, A. I., Roumeliotis, S. I., Johnson, A. E., and Montgomery, J. (2007). Vision-aided inertial navigation for pin-point landing using observations of mapped landmarks. Journal of Field Robotics, 24, 357-378.

Valette, F., Ruffier, F., Viollet, S., and Seidl, T. (2010). Biomimetic optic flow sensing applied to a lunar landing scenario. In the proceedings of the IEEE International Conference on Robotics and Automation (ICRA 2010), pages 2253-2260, Anchorage, Alaska.

Viollet, S. and Franceschini, N. (1999a). Biologically-inspired visual scanning sensor for stabilization and tracking. In Proceedings of the IEEE/RSJ International Conference on Intelligent Robots and Systems.

Viollet, S. and Franceschini, N. (1999b). Visual servo system based on a biologically inspired scanning sensor. In Sensor Fusion and Decentralized control in Robotics II, volume 3839, pages 144-155. SPIE.

Viollet, S. and Franceschini, N. (2001). Super-accurate visual control of an aerial minirobot. In J. S. U. Rckert and U. W. (Eds), editors, Autonomous Minirobots for Research and Edutainment AMIRE, number ISBN 3-93543306-9, pages 215-224, Padderborn, Germany. Heinz Nixdorf Institute.

Wagner, H. (1986). Flight performance and visual control of flight of the free-flying housefly (musca domestica 1.) i. organization of the flight motor. Philosophical Transactions of the Royal Society of London. Series B, Biological Sciences, 312, 527-551.

Watanabe, Y., Fabiani, P., and Le Besnerais, G. (2009). Simultaneous visual target tracking and navigation in a gps-denied environment. In the proceedings of the International Conference on Advanced Robotics (ICAR), pages $1-6$, Munich, Germany.

Watanabe, Y., Lesire, C., Piquereau, A., Fabiani, P., Sanfourche, M., and Le Besnerais, G. (2010). The ONERA ReSSAC unmanned autonomous helicopter: Visual air-to-ground target tracking in an urban environment. In the proceedings of the American Helicopter Society 66th Annual Forum, Phoenix, AZ, USA. 